\title{
A remark on the existence and multiplicity result for a nonlinear elliptic problem involving the p-Laplacian
}

\author{
G. A. Afrouzi and S. H. Rasouli
}

Abstract. In this work, motivated by Wu (J Math Anal Appl 318:253-270, 2006), and using recent ideas from Brown and $\mathrm{Wu}$ (J Math Anal Appl 337:1326-1336, 2008), we prove the existence of nontrivial nonnegative solutions to the following nonlinear elliptic problem:

$$
\begin{cases}-\Delta_{p} u+m(x) u^{p-1}=\lambda a(x) u^{\alpha-1}+b(x) u^{\beta-1}, & x \in \Omega, \\ u=0, & x \in \partial \Omega .\end{cases}
$$

Here $\Delta_{p}$ denotes the p-Laplacian operator defined by $\Delta_{p} z=\operatorname{div}\left(|\nabla z|^{p-2} \nabla z\right)$, $p>2, \Omega \subset \mathbb{R}^{N}$ is a bounded domain with smooth boundary, $2<\beta<p<$ $\alpha<p *\left(p *=\frac{p N}{N-p}\right.$ if $N>p, p *=\infty$ if $\left.N \leq p\right), \lambda \in \mathbb{R} \backslash\{0\}$, the weight $m(x)$ is a bounded function with $\|m\|_{\infty}>0$ and $a(x), b(x)$ are continuous functions which change sign in $\bar{\Omega}$.

Mathematics Subject Classification (2000). 35J50, 35J55, 35J65.

Keywords. Nonlinear elliptic problem, p-Laplacian, Critical points, Nehari manifold.

\section{Introduction}

We are concerned with the existence and multiplicity of nontrivial nonnegative solutions to the nonlinear elliptic problem:

$$
\begin{cases}-\Delta_{p} u+m(x) u^{p-1}=\lambda a(x) u^{\alpha-1}+b(x) u^{\beta-1}, & x \in \Omega, \\ u=0, & x \in \partial \Omega,\end{cases}
$$

where $\Delta_{p}$ denotes the p-Laplacian operator defined by $\Delta_{p} z=\operatorname{div}\left(|\nabla z|^{p-2} \nabla z\right)$, $p>2, \Omega \subset \mathbb{R}^{N}$ is a bounded domain with smooth boundary, $2<\beta<p<\alpha<p *$ $\left(p *=\frac{p N}{N-p}\right.$ if $N>p, p *=\infty$ if $\left.N \leq p\right), \lambda \in \mathbb{R} \backslash\{0\}$, the weight $m(x)$ is a 
bounded function with $\|m\|_{\infty}>0$ and $a(x), b(x) \in C(\bar{\Omega})$ are satisfying $a^{ \pm}=$ $\max \{ \pm a, 0\} \not \equiv 0$ and $b^{ \pm}=\max \{ \pm c, 0\} \not \equiv 0$.

Problems involving the "p-Laplacian" arise from many branches of pure mathematics as in the theory of quasiregular and quasiconformal mapping (see $[13,19])$ as well as from various problems in mathematical physics notably the flow of non-Newtonian fluids: pseudo-plastic fluids correspond to $p \in(1,2)$ while dilatant fluids correspond to $p>2$. The case $p=2$ expresses Newtonian fluids [6]. Especially, quasilinear elliptic promles like (1) arises in the studies of population biology of one species. Here, $u$ is the population density, $-m(x) u^{p-1}+$ $\lambda a(x) u^{\alpha-1}+b(x) u^{\beta-1}$ represents the logistic growth (see [15]).

We are motivated by the paper of $\mathrm{Wu}[20]$, in which problem (1) was discussed when $m \equiv 1, b \equiv 1, p=2$, and $1<\alpha<2<\beta<2^{*}$. The author have altogether proved that, there exists $\lambda_{0}>0$ such that if the parameter $\lambda$ satisfy $0<\lambda<\lambda_{0}$, then problem (1) for $m \equiv 1, b \equiv 1, p=2$, and $1<\alpha<2<\beta<2^{*}$, has at least two positive solutions. In this paper, using the technique of Brown and $\mathrm{Wu}[10]$, we discuss the problem (1) again but with $m \neq \equiv 1, b \neq 1, p>2$, and $2<\beta<p<\alpha<p^{*}$. The change in $\alpha$ completely changes the nature of the solution set of (1). In fact, we shall prove that the problem (1) has at least two solutions $u_{0}^{+}$and $u_{0}^{-}$such that $u_{0}^{ \pm} \geq 0$ in $\Omega$ and $u_{0}^{ \pm} \neq 0$ when the parameter $\lambda$ belongs to a certain subset of $\mathbb{R}$.

In the case when $p=2$, similar problems (with Dirichlet or Neuman boundary condition) have been studied by Binding et al. [7], Ambrosetti et al. [4], and Tehrani $[17,18]$ using variational methods and by Amman and Lopez-Gomez [5] by using global bifurcation theory. Similar problem in the ODE case (semilinear or quasilinear) have been studied in $[2,14]$. We refer to $[3,16]$ for additional results on elliptic problems involving the $p$-Laplacian.

In recent years, several authors use the Nehari manifold to solve semilinear and quasilinear problems (see [1,8-11,20-22]). Brown and Zhang [11] have studied the following subcritical semilinear elliptic equation with a sign-changing weight function

$$
\begin{cases}-\Delta u(x)=\lambda a(x) u+b(x)|u(x)|^{\gamma-2} u(x), & x \in \Omega, \\ u(x)=0, & x \in \partial \Omega,\end{cases}
$$

where $\gamma>2$. Exploiting the relationship between the Nehari manifold and fibering maps [12] (i.e., maps of the form $t \longmapsto J_{\lambda}(t u)$ where $J_{\lambda}$ is the Euler function associated with the equation), they gave an interesting explanation of the wellknown bifurcation result. In fact, the nature of the Nehari manifold changes as the parameter $\lambda$ crosses the bifurcation value. Recently, in [8], the author considered the above problem with $a \equiv 1$ and $1<\gamma<2$. In this work, we give a variational method which is similar to the fibering method (see [12] or [11]) to prove the existence of at least two nontrivial nonnegative solutions of problem (1). In particular, by using the method of [10], we do this without the extraction of the Plais-Smale sequences in the Nehari manifold as in $[1,20]$. 
This paper is divided into three sections, organized as follows. In Sect. 2, we give some notation, preliminaries, properties of the Nehari manifold and set up the variational framework of the problem. In Sect. 3, we give our main result.

\section{Variational setting}

Let $W_{0}^{1, s}=W_{0}^{1, s}(\Omega), s>2$, denote the usual Sobolev space. In the Banach space $W_{0}^{1, p}(\Omega)=W$ we introduce the norm

$$
\|u\|_{W}=\left(\int_{\Omega}\left(|\nabla u|^{p}+m(x)|u|^{p}\right) d x\right)^{\frac{1}{p}} .
$$

which is equivalent to the standard one. First we give the definition of the weak solution of Eq. (1).

Definition 2.1. We say that $u \in W$ is a weak solution to (1) if for any $v \in W$ we have

$\int_{\Omega}\left(|\nabla u|^{p-2} \nabla u . \nabla v+m(x)|u|^{p-2} u v\right) d x=\lambda \int_{\Omega} a(x)|u|^{\alpha-2} u v d x+\int_{\Omega} b(x)|u|^{\beta-2} u v d s$.

It is clear that Problem (1) has a variational structure. Let $\mathcal{J}_{\lambda}: W \rightarrow R$ be the corresponding energy functional of problem (1) is defined by

$$
\mathcal{J}_{\lambda}(u)=\frac{1}{p} M(u)-\frac{1}{\alpha} A(u)-\frac{1}{\beta} B(u),
$$

where

$$
M(u)=\int_{\Omega}\left(|\nabla u|^{p}+m(x)|u|^{p}\right) d x, \quad A(u)=\lambda \int_{\Omega} a(x)|u|^{\alpha} d x
$$

and

$$
B(u)=\int_{\Omega} b(x)|u|^{\beta} d s .
$$

It is well known the weak solutions of Eq. (1) are the critical points of the energy functional $\mathcal{J}_{\lambda}$. Let $I$ be the energy functional associated with an elliptic problem on a Banach space $X$. If $I$ is bounded below and $I$ has a minimizer on $X$, then this minimizer is a critical point of $I$. So, it is a solution of the corresponding elliptic problem. However, the energy functional $\mathcal{J}_{\lambda}$, is not bounded below on the whole space $W$, but is bounded on an appropriate subset, and a minimizer on this set (if it exists) gives rise to solution to Eq. (1).

Consider the Nehari minimization problem for $\lambda \in \mathbb{R} \backslash\{0\}$,

$$
\gamma_{\lambda}=\inf \left\{\mathcal{J}_{\lambda}(u): u \in \mathcal{N}_{\lambda}\right\},
$$

where $\mathcal{N}_{\lambda}=\left\{u \in W \backslash\{0\}:\left\langle\mathcal{J}_{\lambda}^{\prime}(u), u\right\rangle=0\right\}$. It is easy to see that $u \in \mathcal{N}_{\lambda}$ if and only if

$$
M(u)-A(u)=B(u)
$$


Note that $\mathcal{N}_{\lambda}$ contains every nonzero solution of problem (1). Define

$$
\mathcal{G}_{\lambda}(u)=\left\langle\mathcal{J}_{\lambda}^{\prime}(u), u\right\rangle
$$

Then for $u \in N_{\lambda}$,

$$
\begin{aligned}
\left\langle\mathcal{G}_{\lambda}^{\prime}(u), u\right\rangle & =p M(u)-\alpha A(u)-\beta B(u) \\
& =(p-\alpha) A(u)-(\beta-p) B(u) \\
& =(p-\alpha) M(u)-(\beta-\alpha) B(u) \\
& =(p-\beta) M(u)-(\alpha-\beta) A(u) .
\end{aligned}
$$

Now, we split $\mathcal{N}_{\lambda}$ into three parts:

$$
\begin{gathered}
\mathcal{N}_{\lambda}^{+}=\left\{u \in N_{\lambda}:\left\langle\mathcal{G}_{\lambda}^{\prime}(u), u\right\rangle>0\right\}, \\
\mathcal{N}_{\lambda}^{0}=\left\{u \in N_{\lambda}:\left\langle\mathcal{G}_{\lambda}^{\prime}(u), u\right\rangle=0\right\}, \\
\mathcal{N}_{\lambda}^{-}=\left\{u \in N_{\lambda}:\left\langle\mathcal{G}_{\lambda}^{\prime}(u), u\right\rangle<0\right\} .
\end{gathered}
$$

To state our main result, we now present some important properties of $\mathcal{N}_{\lambda}^{+}$, $\mathcal{N}_{\lambda}^{0}$, and $\mathcal{N}_{\lambda}^{-}$.

Lemma 2.2. There exists $\delta_{0}>0$ such that for $0<\lambda\|a\|_{\infty}<\delta_{0}$, we have $\mathcal{N}_{\lambda}^{0}=\emptyset$.

Proof. Suppose otherwise, then for $\delta_{0}=\left[\frac{\alpha-p}{(\alpha-\beta) C_{2}^{\beta}\|b\|_{\infty}}\right]^{\frac{\alpha-\beta}{p-\beta}}\left[\frac{p-\beta}{(\alpha-\beta) C_{1}^{\alpha}}\right]$, where $C_{1}, C_{2}$ are positive constant and specified later, there exists $\lambda$ with $0<\lambda\|a\|_{\infty}<$ $\delta_{0}$ such that $\mathcal{N}_{\lambda}^{0} \neq \emptyset$. Then for $u \in \mathcal{N}_{\lambda}^{0}$ we have

$$
\begin{aligned}
0 & =\left\langle\mathcal{G}_{\lambda}^{\prime}(u), u\right\rangle=(p-\beta) M(u)+(\beta-\alpha) A(u) \\
& =(p-\alpha) M(u)+(\alpha-\beta) B(u) .
\end{aligned}
$$

By the Sobolev imbedding theorem,

$$
A(u) \leq \lambda\|a\|_{\infty}\|u\|_{\alpha}^{\alpha} \leq \lambda C_{1}^{\alpha}\|a\|_{\infty}\|u\|_{W}^{\alpha},
$$

and

$$
B(u) \leq\|b\|_{\infty}\|u\|_{\beta}^{\beta} \leq C_{2}^{\beta}\|b\|_{\infty}\|u\|_{W}^{\beta} .
$$

By using (9)-(10) in (7)-(8) we get

$$
\|u\|_{W} \geq\left(\frac{p-\beta}{\alpha-\beta}\right)^{\frac{1}{\alpha-p}}\left(\frac{1}{C_{1}^{\alpha} \lambda\|a\|_{\infty}}\right)^{\frac{1}{\alpha-p}}
$$

and

$$
\|u\|_{W} \leq\left(\frac{(\beta-\beta)}{\alpha-p}\right)^{\frac{1}{p-\beta}}\left(C_{2}^{\beta}\|b\|_{\infty}\right)^{\frac{1}{p-\beta}} .
$$

This implies $\lambda\|a\|_{\infty} \geq \delta_{0}$, which is a contradiction. Thus, we can conclude that there exists $\delta_{0}>0$ such that for $0<\lambda\|a\|_{\infty}<\delta_{0}$, we have $\mathcal{N}_{\lambda}^{0}=\emptyset$. 
By Lemma 2.2, for $0<\lambda\|a\|_{\infty}<\delta_{0}$ we write $\mathcal{N}_{\lambda}=\mathcal{N}_{\lambda}^{+} \cup \mathcal{N}_{\lambda}^{-}$and define

$$
\gamma_{\lambda}^{+}=\inf _{u \in \mathcal{N}_{\lambda}^{+}} \mathcal{J}_{\lambda}(u) ; \gamma_{\lambda}^{-}=\inf _{u \in \mathcal{N}_{\lambda}^{-}} \mathcal{J}_{\lambda}(u)
$$

Lemma 2.3. We have

(i) If $u \in \mathcal{N}_{\lambda}^{+}$, then $B(u)>0$;

(ii) If $u \in \mathcal{N}_{\lambda}^{-}$, then $A(u)>0$.

Proof. (i) We consider the following two cases:

Case (i-a): $A(u)=0$. We have

$$
B(u)=M(u)>0 .
$$

Case (i-b): $A(u) \neq 0$. Since $(u) \in \mathcal{N}_{\lambda}^{+}$, by (5), we have

$$
(p-\alpha) M(u)+(\alpha-\beta) B(u)>0,
$$

which implies

$$
B(u)>\frac{\alpha-p}{\alpha-\beta} M(u)>0 .
$$

(ii) We consider the following two cases:

Case (ii-a): $B(u) \leq 0$, we have

$$
A(u)=M(u)-B(u)>0 .
$$

Case (ii-b): $B(u)>0$. By (4), we have

$$
(p-\alpha) A(u)+(p-\beta) B(u)<0,
$$

which implies

$$
A(u)>\frac{p--\beta}{\alpha-p} B(u)>0 .
$$

It follows that the conclusion is true.

Also, as proved in Binding et al. [7] or in Brown and Zhang [11], we have the following lemma.

Lemma 2.4. Suppose that $u_{0}$ is a local minimizer for $\mathcal{J}_{\lambda}$ on $\mathcal{N}_{\lambda}$. Then, if $u_{0} \notin \mathcal{N}_{\lambda}^{0}$, $u_{0}$ is a critical point of $\mathcal{J}_{\lambda}$.

Proof. If $u_{0}$ is a local minimizer for $\mathcal{J}_{\lambda}$ on $\mathcal{N}_{\lambda}$, then $u_{0}$ is a solution of the optimization problem

$$
\text { minimize } \mathcal{J}_{\lambda}(u) \text { subject to } \mathcal{G}_{\lambda}(u)=0 \text {. }
$$

Hence, by the theory of Lagrange multipliers, there exists $\Lambda \in \mathbb{R}$ such that

$$
\mathcal{J}_{\lambda}^{\prime}\left(u_{0}\right)=\Lambda \mathcal{G}_{\lambda}^{\prime}\left(u_{0}\right) \quad \text { in } W^{-1}(\Omega) .
$$

Here $W^{-1}(\Omega)$ is the dual space of the Soboleve space $W$. Thus,

$$
\left\langle\mathcal{J}_{\lambda}^{\prime}(u), u\right\rangle_{W}=\Lambda\left\langle\mathcal{G}_{\lambda, \mu}^{\prime}(u), u\right\rangle_{W}
$$



proof.

But $\left\langle\mathcal{G}_{\lambda}^{\prime}(u), u\right\rangle_{W} \neq 0$, since $u_{0} \notin \mathcal{N}_{\lambda}^{0}$. Hence $\Lambda=0$. This completes the

Then we have the following result.

Lemma 2.5. $\mathcal{J}_{\lambda}$ is coercive and bounded below on $N_{\lambda}$.

Proof. If $u \in \mathcal{N}_{\lambda}$, it follows from (2) and the Sobolev embedding theorem

$$
\begin{aligned}
\mathcal{J}_{\lambda}(u) & =\left(\frac{\alpha-p}{\alpha p}\right) M(u)-\left(\frac{\alpha-\beta}{\alpha \beta}\right) B(u) \\
& \geq\left(\frac{\alpha-p}{\alpha p}\right) M(u)-\left(\frac{\alpha-\beta}{\alpha \beta}\right) C_{2}^{\beta}\|b\|_{\infty}\|u\|_{W}^{\beta} \\
& =\left(\frac{\alpha-p}{\alpha p}\right) M(u)-\left(\frac{\alpha-\beta}{\alpha \beta}\right) C_{2}^{\beta}\|b\|_{\infty}(M(u))^{\beta / p} .
\end{aligned}
$$

Thus $\mathcal{J}_{\lambda}$ is coercive and bounded below on $N_{\lambda}$.

Lemma 2.6. Let $\delta^{*}=\left(\frac{\beta}{p}\right)^{\frac{\alpha-p}{p-\beta}} \delta_{0}$. Then if $0<\lambda\|a\|_{\infty}<\delta^{*}$, We have

(i) $\gamma_{\lambda}^{+}<0$;

(ii) $\gamma_{\lambda}^{-} \geq k_{0}$, for some $k_{0}=k_{0}\left(\alpha, \beta, C_{1}, C_{2}\right)$.

Proof. (i) Let $u \in \mathcal{N}_{\lambda}^{+}$. By (6)

$$
\frac{p-\beta}{\alpha-\beta} M(u)>A(u),
$$

and so

$$
\begin{aligned}
\mathcal{J}_{\lambda}(u) & =\left(\frac{1}{p}-\frac{1}{\beta}\right) M(u)+\left(\frac{1}{\beta}-\frac{1}{\alpha}\right) A(u) \\
& \leq\left(\frac{\beta-p}{p \beta}\right) M(u)+\left(\frac{\alpha-\beta}{\alpha \beta}\right)\left[\frac{(p-\beta)}{(\alpha-\beta)} M(u)\right] \\
& =\left[\frac{\beta-p}{p \beta}+\frac{p-\beta}{\alpha \beta}\right] M(u) \\
& =\frac{(\alpha-p)(\beta-p)}{p \alpha \beta} M(u)<0 .
\end{aligned}
$$

Thus $\gamma_{\lambda}^{+}<0$.

(ii) Let $u \in \mathcal{N}_{\lambda}^{-}$, by (6) and (9) we have

$$
M(u)<\frac{\alpha-\beta}{p-\beta} A(u) \leq \frac{\alpha-\beta}{p-\beta} C_{1}^{\alpha}\left(\lambda\|a\|_{\infty}\right)\|u\|_{W}^{\alpha} .
$$

This implies

$$
\|u\|_{W}>\left[\frac{p-\beta}{(\alpha-\beta) C_{1}^{\alpha}\left(\lambda\|a\|_{\infty}\right)}\right]^{\frac{1}{\alpha-p}} \quad \text { for all } u \in \mathcal{N}_{\lambda}^{-} .
$$


By Lemma 2.5, we have

$$
\begin{aligned}
\mathcal{J}_{\lambda}(u) \geq & \|u\|_{W}^{\beta}\left[\left(\frac{\alpha-p}{\alpha p}\right)\|u\|_{W}^{p-\beta}-\left(\frac{\alpha-\beta}{\alpha \beta}\right) C_{2}^{\beta}\|b\|_{\infty}\right] \\
> & \left(\frac{p-\beta}{(\alpha-\beta) C_{1}^{\alpha}\left(\lambda\|a\|_{\infty}\right)}\right)^{\frac{\beta}{\alpha-p}}\left[\left(\frac{\alpha-p}{\alpha p}\right)\left(\frac{p-\beta}{(\alpha-\beta) C_{1}^{\alpha}\left(\lambda\|a\|_{\infty}\right)}\right)^{\frac{p-\beta}{\alpha-p}}\right. \\
& \left.-\left(\frac{\alpha-\beta}{\alpha \beta}\right) C_{2}^{\beta}\|b\|_{\infty}\right] .
\end{aligned}
$$

Thus, if

$$
0<\lambda\|a\|_{\infty}<\delta^{*}
$$

then

$$
\mathcal{J}_{\lambda}>k_{0}, \quad \text { for all } u \in \mathcal{N}_{\lambda}^{-},
$$

for some $k_{0}=k_{0}\left(\alpha, \beta, C_{1}, C_{2}\right)>0$. This completes the proof.

For each $u \in W$ with $A(u)>0$, we write

$$
t_{\max }=\left(\frac{(p-\beta) M(u)}{(\alpha-\beta) A(u)}\right)^{1 /(\alpha-p)}>0 .
$$

Then we have the following lemma.

Lemma 2.7. For each $u \in W$ with $A(u)>0$ and $0<\lambda\|a\|_{\infty}<\delta_{0}$, we have

(i) if $B(u) \leq 0$, then there is unique $t^{-}>t_{\max }$ such that $t^{-} u \in \mathcal{N}_{\lambda}^{-}$and

$$
\mathcal{J}_{\lambda}\left(t^{-} u\right)=\sup _{t \geq 0} \mathcal{J}_{\lambda}(t u)
$$

(ii) if $B(u)>0$, then there are unique $0<t^{+}=t^{+}(u)<t_{\max }<t^{-}$such that $t^{+} u \in \mathcal{N}_{\lambda}^{+}, t^{-} u \in \mathcal{N}_{\lambda}^{-}$and

$$
\mathcal{J}_{\lambda}\left(t^{+} u\right)=\inf _{0 \leq t \leq t_{\max }} \mathcal{J}_{\lambda}(t u), \mathcal{J}_{\lambda}\left(t^{-} u\right)=\sup _{t \geq 0} \mathcal{J}_{\lambda}(t u) .
$$

Proof. Fix $u \in W$ with $A(u)>0$. Let

$$
E(t)=t^{p-\beta} M(u)-t^{\alpha-\beta} A(u) \quad \text { for } t \geq 0 .
$$

Clearly, $E(0)=0, E(t) \rightarrow-\infty$ as $t \rightarrow \infty$. Since

$$
E^{\prime}(t)=(p-\beta) t^{p-\beta-1} M(u)-(\alpha-\beta) t^{\alpha-\beta-1} A(u),
$$


we have $E^{\prime}(t)=0$ at $t=t_{\max }, E^{\prime}(t)>0$ for $t \in\left[0, t_{\max }\right)$ and $E^{\prime}(t)<0$ for $t \in\left(t_{\max }, \infty\right)$. Then $E(t)$ achieves its maximum at $t_{\max }$, increasing for $t \in\left[0, t_{\max }\right)$ and decreasing for $t \in\left(t_{\max }, \infty\right)$. Moreover,

$$
\begin{aligned}
E\left(t_{\max }\right) & =\left(\frac{(p-\beta) M(u)}{(\alpha-\beta) A(u)}\right)^{\frac{p-\beta}{\alpha-p}} M(u)-\left(\frac{(p-\beta) M(u)}{(\alpha-\beta) A(u)}\right)^{\frac{\alpha-\beta}{\alpha-p}} A(u) \\
& =\|u\|_{W}^{\beta}\left[\left(\frac{p-\beta}{\alpha-\beta}\right)^{\frac{p-\beta}{\alpha-p}}-\left(\frac{p-\beta}{\alpha-\beta}\right)^{\frac{\alpha-\beta}{\alpha-p}}\right]\left(\frac{\|u\|_{W}^{\alpha}}{A(u)}\right)^{\frac{p-\beta}{\alpha-p}} \\
& \geq\|u\|_{W}^{\beta}\left(\frac{1}{\lambda\|a\|_{\infty}}\right)^{\frac{p-\beta}{\alpha-\beta}}\left(\frac{\alpha-p}{\alpha-\beta}\right)\left(\frac{p-\beta}{(\alpha-\beta) C_{1}^{\alpha}}\right)^{\frac{p-\beta}{\alpha-\beta}} .
\end{aligned}
$$

(i) $B(u) \leq 0$ : There is a unique $t^{-}>t_{\max }$ such that $E\left(t^{-}\right)=\lambda B(u)$ and $E^{\prime}\left(t^{-}\right)<0$. Now,

$$
\begin{aligned}
(p-\beta) M\left(t^{-} u\right)-(\alpha-\beta) A\left(t^{-} u\right)= & \left(t^{-}\right)^{1+\beta}\left[(p-\beta)\left(t^{-}\right)^{p-1-\beta} M(u)\right. \\
& \left.-(\alpha-\beta)\left(t^{-}\right)^{\alpha-\beta-1} A(u)\right] \\
= & \left(t^{-}\right)^{1+\beta} E^{\prime}\left(t^{-}\right)<0,
\end{aligned}
$$

and

$$
\begin{aligned}
\left\langle\mathcal{J}_{\lambda}^{\prime}\left(t^{-} u\right), t^{-} u\right\rangle & =\left(t^{-}\right)^{p} M(u)-\left(t^{-}\right)^{\alpha} A(u)-\left(t^{-}\right)^{\beta} B(u) \\
& =\left(t^{-}\right)^{\beta}\left[\left(t^{-}\right)^{p-\beta} M(u)-\left(t^{-}\right)^{\alpha-\beta} A(u)-B(u)\right] \\
& =\left(t^{-}\right)^{\beta}\left[E\left(t^{-}\right)-B(u)\right]=0 .
\end{aligned}
$$

Thus, $t^{-} u \in \mathcal{N}_{\lambda}^{-}$. Since for $t>t_{\max }$, we have

$$
\begin{aligned}
& (p-\beta) M(t u)-(\alpha-\beta) A(t u)<0, \\
& \frac{d^{2}}{d t^{2}} \mathcal{J}_{\lambda}(t u)<0
\end{aligned}
$$

and

$$
\frac{d}{d t} \mathcal{J}_{\lambda}(t u)=t^{p-1} M(u)-t^{\alpha-1} A(u)-t^{\beta-1} B(u)=0 \quad \text { for } t=t^{-}
$$

Thus, $\mathcal{J}_{\lambda}\left(t^{-} u\right)=\sup _{t \geq 0} \mathcal{J}_{\lambda}(t u)$.

(ii) $B(u)>0$. By (13) and

$$
\begin{aligned}
E(0) & =0<B(u) \\
& \leq C_{2}^{\beta}\|b\|_{\infty}\|u\|_{W}^{\beta} \\
& <\|u\|_{W}^{\beta}\left(\frac{1}{\lambda\|a\|_{\infty}}\right)^{\frac{p-\beta}{\alpha-\beta}}\left(\frac{\alpha-p}{\alpha-\beta}\right)\left(\frac{p-\beta}{(\alpha-\beta) C_{1}^{\alpha}}\right)^{\frac{p-\beta}{\alpha-\beta}} \\
& \leq E\left(t_{\max }\right)
\end{aligned}
$$


for $0<\lambda\|a\|_{\infty}<\delta_{0}$, there are unique $t^{+}$and $t^{-}$such that $0<t^{+}<t_{\max }<t^{-}$,

$$
\begin{aligned}
& E\left(t^{+}\right)=B(u)=E\left(t^{-}\right), \\
& E^{\prime}\left(t^{+}\right)>0>E^{\prime}\left(t^{-}\right) .
\end{aligned}
$$

We have $t^{+} u \in \mathcal{N}_{\lambda}^{+}, t^{-} u \in \mathcal{N}_{\lambda}^{-}$, and $\mathcal{J}_{\lambda}\left(t^{-} u\right) \geq \mathcal{J}_{\lambda}(t u) \geq \mathcal{J}_{\lambda}\left(t^{+} u\right)$ for each $t \in\left[t^{+}, t^{-}\right]$and $\mathcal{J}_{\lambda}\left(t^{+} u\right) \leq \mathcal{J}_{\lambda}(t u)$ for each $t \in\left[0, t^{+}\right]$. Thus,

$$
\mathcal{J}_{\lambda}\left(t^{+} u\right)=\inf _{0 \leq t \leq t_{\max }} \mathcal{J}_{\lambda}(t u), \mathcal{J}_{\lambda}\left(t^{-} u\right)=\sup _{t \geq 0} \mathcal{J}_{\lambda}(t u) .
$$

This completes the proof.

For each $u \in W$ with $B(u)>0$, we write

$$
\bar{t}_{\max }=\left(\frac{(\alpha-\beta) B(u)}{(\alpha-p) M(u)}\right)^{1 /(p-\beta)}>0 .
$$

Then we have the following lemma.

Lemma 2.8. For each $u \in W$ with $B(u)>0$, we have

(i) if $A(u) \leq 0$, then there is unique $t^{+}<\bar{t}_{\max }$ such that $t^{+} u \in \mathcal{N}_{\lambda}^{+}$and

$$
\mathcal{J}_{\lambda}\left(t^{+} u\right)=\inf _{t \geq 0} \mathcal{J}_{\lambda}(t u)
$$

(ii) if $A(u)>0$, then there are unique $0<t^{+}=t^{+}(u)<\bar{t}_{\max }<t^{-}$such that $t^{+} u \in \mathcal{N}_{\lambda}^{+}, t^{-} u \in \mathcal{N}_{\lambda}^{-}$and

$$
\mathcal{J}_{\lambda}\left(t^{+} u\right)=\inf _{0 \leq t \leq \bar{t}_{\max }} \mathcal{J}_{\lambda}(t u), \mathcal{J}_{\lambda}\left(t^{-} u\right)=\sup _{t \geq 0} \mathcal{J}_{\lambda}(t u)
$$

Proof. Fix $u \in W$ with $B(u)>0$. Let

$$
\bar{E}(t)=t^{p-\alpha} M(u)-t^{\beta-\alpha} B(u) \text { for } t>0 .
$$

Clearly, $\bar{E}(t) \rightarrow-\infty$ as $t \rightarrow 0^{+}$. Since

$$
\bar{E}^{\prime}(t)=(p-\alpha) t^{p-\alpha-1} M(u)-(\beta-\alpha) t^{\beta-\alpha-1} B(u),
$$

we have $\bar{E}^{\prime}(t)=0$ at $t=\bar{t}_{\max }, \bar{E}^{\prime}(t)>0$ for $t \in\left[0, \bar{t}_{\max }\right)$ and $\bar{E}^{\prime}(t)<0$ for $t \in\left(\bar{t}_{\text {max }}, \infty\right)$. Then $\bar{E}(t)$ achieves its maximum at $\bar{t}_{\text {max }}$, increasing for $t \in\left[0, \bar{t}_{\text {max }}\right)$ and decreasing for $t \in\left(\bar{t}_{\max }, \infty\right)$. Similar to argument in Lemma 2.7, we can obtain the result of Lemma 2.8 . 


\section{Existence of solutions}

Now we can state our main result.

Theorem 3.1. If the parameter $\lambda$ satisfy $0<\lambda\|a\|_{\infty}<\delta^{*}$, then problem (1) has at least two solutions $u_{0}^{+}$and $u_{0}^{-}$such that $u_{0}^{ \pm} \geq 0$ in $\Omega$ and $u_{0}^{ \pm} \neq 0$.

The proof of this Theorem will be a consequence of the next two propositions.

Proposition 3.2. If $0<\lambda\|a\|_{\infty}<\delta^{*}$, then the functional $\mathcal{J}_{\lambda}$ has a minimizer $u_{0}^{+}$ in $\mathcal{N}_{\lambda}^{+}$and it satisfies

(i) $\mathcal{J}_{\lambda}\left(u_{0}^{+}\right)=\gamma_{\lambda}^{+}$;

(ii) $u_{0}^{+}$is a nontrivial nonnegative solution of problem (1), such that $u_{0}^{+} \geq 0$ in $\Omega$ and $u_{0}^{+} \neq 0$.

Proof. By Lemma 2.5, $\mathcal{J}_{\lambda}$ is coercive and bounded below on $\mathcal{N}_{\lambda}$. Let $\left\{u_{n}\right\}$ be a minimizing sequence for $\mathcal{J}_{\lambda}$ on $\mathcal{N}_{\lambda}^{+}$, i.e., $\lim _{n \rightarrow \infty} \mathcal{J}_{\lambda}\left(u_{n}\right)=\inf _{u \in \mathcal{N}_{\lambda}^{+}} \mathcal{J}_{\lambda}(u)$. Then by Lemma 2.5 and the Rellich-Kondrachov theorem, there exist a subsequence $\left\{u_{n}\right\}$ and $u_{0}^{+} \in W$ such that $u_{0}^{+}$is a solution of problem (1) and

$$
\begin{aligned}
& u_{n} \rightarrow u_{0}^{+} \quad \text { weakly in } W, \\
& u_{n} \rightarrow u_{0}^{+} \quad \text { strongly in } L^{\alpha}(\Omega) \text { and in } L^{\beta}(\Omega) .
\end{aligned}
$$

This implies

$$
\begin{gathered}
B\left(u_{n}\right) \rightarrow B\left(u_{0}^{+}\right) \quad \text { as } n \rightarrow \infty \\
A\left(u_{n}\right) \rightarrow A\left(u_{0}^{+}\right) \quad \text { as } n \rightarrow \infty
\end{gathered}
$$

Since

$$
\mathcal{J}_{\lambda}\left(u_{n}\right)=\left(\frac{\alpha-p}{\alpha p}\right) M\left(u_{n}\right)-\left(\frac{\alpha-\beta}{\alpha \beta}\right) B\left(u_{n}\right),
$$

and by Theorem 2.6(i)

$$
\mathcal{J}_{\lambda}\left(u_{n}\right) \rightarrow \gamma_{\lambda}^{+}<0 \quad \text { as } n \rightarrow \infty \text {. }
$$

Letting $n \rightarrow \infty$, we see that $B\left(u_{0}\right)>0$. In particular $u_{0}^{+} \neq 0$. Now we prove that $u_{n} \rightarrow u_{0}^{+}$strongly in $W$. Suppose otherwise, then

$$
\left\|u_{0}^{+}\right\|_{W}<\liminf _{n \rightarrow \infty}\left\|u_{n}\right\|_{W} .
$$

Fix $u \in W$ with $B(u)>0$. Let

$$
K_{u}(t)=\bar{E}(t)-A(u),
$$

where $\bar{E}(t)$ is as in (15.) Clearly, $K_{u}(t) \rightarrow-\infty$ as $t \rightarrow 0^{+}$, and

$$
K_{u}(t) \rightarrow-A(u) \quad \text { as } t \rightarrow \infty .
$$


Since $K_{u}^{\prime}(t)=\bar{E}^{\prime}(t)$, similar argument as in the proof of Lemma 2.8, we have $K_{u}(t)$ achieves its maximum at $\bar{t}_{\text {max }}$, is increasing for $t \in\left(0, \bar{t}_{\text {max }}\right)$ and decreasing for $t \in\left(\bar{t}_{\text {max }}, \infty\right)$, where

$$
\bar{t}_{\max }=\left(\frac{(\alpha-\beta) B(u)}{(\alpha-p) M(u)}\right)^{1 /(p-\beta)}>0,
$$

is as in (14). Since $B\left(u_{0}^{+}\right)>0$, by Lemma 2.8, there is unique $t_{0}^{+}<\bar{t}_{\max }$ such that $t_{0}^{+} u_{0}^{+} \in \mathcal{N}_{\lambda}^{+}$and

$$
\mathcal{J}_{\lambda}\left(t_{0}^{+} u_{0}^{+}\right)=\inf _{0 \leq t \leq \bar{t}_{\max }\left(u_{0}\right)} \mathcal{J}_{\lambda}\left(t u_{0}^{+}\right) .
$$

Then

$$
\begin{aligned}
K_{u_{0}^{+}}\left(t_{0}^{+}\right) & =\left(t_{0}^{+}\right)^{p-\alpha} M\left(u_{0}^{+}\right)-\left(t_{0}^{+}\right)^{\beta-\alpha} B\left(u_{0}^{+}\right)-A\left(u_{0}^{+}\right) \\
& =\left(t_{0}^{+}\right)^{-\alpha}\left(M\left(t_{0}^{+} u_{0}^{+}\right)-B\left(t_{0}^{+} u_{0}^{+}\right)-A\left(t_{0}^{+} u_{0}^{+}\right)\right) \\
& =0 .
\end{aligned}
$$

By (16) and (17) we obtain

$$
K_{u_{n}}\left(t_{0}^{+}\right)>0 \text { for } \mathrm{n} \text { sufficiently large. }
$$

Since $u_{n} \in \mathcal{N}_{\lambda}^{+}$, we have $\bar{t}_{\max }\left(u_{n}\right)>1$. Moreover,

$$
K_{u_{n}}(1)=M\left(u_{n}\right)-B\left(u_{n}\right)-A\left(u_{n}\right)=0,
$$

and $K_{u_{n}}(t)$ is increasing for $t \in\left(0, \bar{t}_{\max }\left(u_{n}\right)\right)$. This implies $K_{u_{n}}(t)<0$ for all $t \in(0,1]$ and $n$ sufficiently large. We obtain $1<t_{0}^{+} \leq \bar{t}_{\max }\left(u_{0}\right)$. But $t_{0}^{+} u_{0}^{+} \in \mathcal{N}_{\lambda}^{+}$ and

$$
\mathcal{J}_{\lambda}\left(t_{0}^{+} u_{0}^{+}\right)=\inf _{0 \leq t \leq \bar{t}_{\max }\left(u_{0}\right)} \mathcal{J}_{\lambda}\left(t u_{0}^{+}\right)
$$

This implies

$$
\mathcal{J}_{\lambda}\left(t_{0}^{+} u_{0}^{+}\right)<\mathcal{J}_{\lambda}\left(u_{0}^{+}\right)<\lim _{n \rightarrow \infty} \mathcal{J}_{\lambda}\left(u_{n}\right)=\gamma_{\lambda}^{+},
$$

which is a contradiction. Hence

$$
u_{n} \rightarrow u_{0}^{+} \quad \text { strongly in } W \text {. }
$$

This implies

$$
\mathcal{J}_{\lambda}\left(u_{n}\right) \rightarrow \mathcal{J}_{\lambda}\left(u_{0}^{+}\right)=\gamma_{\lambda}^{+} \quad \text { as } n \rightarrow \infty .
$$

Thus $u_{0}^{+}$is a minimizer for $\mathcal{J}_{\lambda}$ on $\mathcal{N}_{\lambda}^{+}$. Since $\mathcal{J}_{\lambda}\left(u_{0}^{+}\right)=\mathcal{J}_{\lambda}\left(\left|u_{0}^{+}\right|\right)$and $\left|u_{0}^{+}\right| \in \mathcal{N}_{\lambda}^{+}$, by Lemma 2.4 we may assume that $u_{0}^{+}$is a nontrivial nonnegative solution of Eq. (1).

Next, we establish the existence of a local minimum for $\mathcal{J}_{\lambda}$ on $\mathcal{N}_{\lambda}^{-}$.

Proposition 3.3. If $0<\lambda\|a\|_{\infty}<\delta^{*}$, then the functional $\mathcal{J}_{\lambda}$ has a minimizer $u_{0}^{-}$ in $\mathcal{N}_{\lambda}^{-}$and it satisfies

(i) $\mathcal{J}_{\lambda}\left(u_{0}^{-}\right)=\gamma_{\lambda}^{-}$; 
(ii) $u_{0}^{-}$is a nontrivial nonnegative solution of problem (1), such that $u_{0}^{-} \geq 0$ in $\Omega$ and $u_{0}^{-} \neq 0$.

Proof. Let $\left\{u_{n}\right\}$ be a minimizing sequence for $\mathcal{J}_{\lambda}$ on $\mathcal{N}_{\lambda}^{-}$, i.e., $\lim _{n \rightarrow \infty} \mathcal{J}_{\lambda}\left(u_{n}\right)=$ $\inf _{u \in \mathcal{N}_{\lambda}^{-}} \mathcal{J}_{\lambda}(u)$. Then by Lemma 2.5 and the Rellich-Kondrachov theorem, there exist a subsequence $\left\{u_{n}\right\}$ and $u_{0}^{-} \in W$ such that $u_{0}^{-}$is a solution of problem (1) and

$$
\begin{aligned}
& u_{n} \rightarrow u_{0}^{-} \quad \text { weakly in } W, \\
& u_{n} \rightarrow u_{0}^{-} \quad \text { strongly in } L^{\alpha}(\Omega) \text { and in } L^{\beta}(\Omega) .
\end{aligned}
$$

This implies

$$
\begin{aligned}
& A\left(u_{n}\right) \rightarrow A\left(u_{0}^{-}\right) \quad \text { as } n \rightarrow \infty \\
& B\left(u_{n}\right) \rightarrow B\left(u_{0}^{-}\right) \quad \text { as } n \rightarrow \infty .
\end{aligned}
$$

Moreover, by (6) we obtain

$$
A\left(u_{n}\right)>\frac{p-\beta}{\alpha-\beta} M\left(u_{n}\right) ;
$$

By (12) and (18) there exists a positive number $\eta_{0}$ such that

$$
A\left(u_{n}\right)>\eta_{0}
$$

This implies

$$
A\left(u_{0}^{-}\right) \geq \eta_{0}
$$

Now we prove that $u_{n} \rightarrow u_{0}^{-}$strongly in $W$. Suppose otherwise, then

$$
\left\|u_{0}^{-}\right\|_{W}<\liminf _{n \rightarrow \infty}\left\|u_{n}\right\|_{W} .
$$

By Lemma 2.7, there is unique $t_{0}^{-}$such that $t_{0}^{-} u_{0}^{-} \in \mathcal{N}_{\lambda}^{-}$. Since $\left\{u_{n}\right\} \in \mathcal{N}_{\lambda}^{-}$, $\mathcal{J}_{\lambda}\left(u_{n}\right) \geq \mathcal{J}_{\lambda}\left(t u_{n}\right)$ for all $t \geq 0$, we have

$$
\mathcal{J}_{\lambda}\left(t_{0}^{-} u_{0}^{-}\right)<\lim _{n \rightarrow \infty} \mathcal{J}_{\lambda}\left(t_{0}^{-} u_{n}\right) \leq \lim _{n \rightarrow \infty} \mathcal{J}_{\lambda}\left(u_{n}\right)=\gamma_{\lambda}^{-},
$$

and this is a contradiction. Hence

$$
u_{n} \rightarrow u_{0}^{-} \quad \text { strongly in } W \text {. }
$$

This implies

$$
\mathcal{J}_{\lambda}\left(u_{n}\right) \rightarrow \mathcal{J}_{\lambda}\left(u_{0}^{-}\right)=\gamma_{\lambda}^{-} \quad \text { as } n \rightarrow \infty .
$$

Since $\mathcal{J}_{\lambda}\left(u_{0}^{-}\right)=\mathcal{J}_{\lambda}\left(\left|u_{0}^{-}\right|\right)$and $\left|u_{0}^{-}\right| \in \mathcal{N}_{\lambda}^{-}$, by Lemma 2.4 and (19) we may assume that $u_{0}^{-}$is a nontrivial nonnegative solution of Eq. (1).

Proof of Theorem 3.1. By Propositions 3.2 and 3.3, we obtain Eq. (1) has two nontrivial nonnegative solutions $u_{0}^{+}$and $u_{0}^{-}$such that $u_{0}^{+} \in \mathcal{N}_{\lambda}^{+}$and $u_{0}^{-} \in \mathcal{N}_{\lambda}^{-}$. It remain to show that the solutions found in Propositions 3.2 and 3.3 are distinct. Since $\mathcal{N}_{\lambda}^{+} \cap \mathcal{N}_{\lambda}^{-}=\emptyset$, this implies that $u_{0}^{+}$and $u_{0}^{-}$are distinct. This concludes the proof. 


\section{References}

[1] Alves, C.O., El Hamidi, A.: Nehari manifold and existence of positive solutions tob a class of quasilinear problems. Nonl. Anal. 60, 611-624 (2005)

[2] Afrouzi, G.A.: On a nonlinear eigenvalue Problem in ODE. J. Math. Anal. Appl. 303, 342-349 (2005)

[3] Afrouzi, G.A., Rasouli, S.H.: A remark on the uniqueness of positive solutions for Dirichlet problems. Nonl. Anal. 64(12), 2773-2777 (2006)

[4] Ambrosetti, A., Brezis, H., Cerami, G.: Combined effects of concave and convex nonlinearities in some elliptic problems. J. Funct. Anal. 122, 519-543 (1994)

[5] Amman, H., Lopez-Gomez, J.: A priori bounds and multiple solution for superlinear indefinite elliptic problems. J. Differ. Equ. 146, 336-374 (1998)

[6] Atkinson, C., El Kalli, K.: Some boundary value problems for the Bingham model. J. Non-Newtonian Fluid Mech. 41, 339-363 (1992)

[7] Binding, P.A., Drabek, P., Huang, Y.X.: On Neuman boundary value problems for some quasilinear equations. Electron. J. Differ. Equ. 5, 1-11 (1997)

[8] Brown, K.J.: The Nehari manifold for a semilinear elliptic equation involving a sublinear term. Calc. Var. 22, 483-494 (2005)

[9] Brown, K.J., Wu, T.F.: A fibering map approach to a semilinear elliptic boundary value problem. Electron. J. Differ. Equ. 69, 1-9 (2007)

[10] Brown, K.J., Wu, T.F.: A semilinear elliptic system involving nonlinear boundary condition and sign changing weight function. J. Math. Anal. Appl. 337, 1326-1336 (2008)

[11] Brown, K.J., Zhang, Y.: The Nehari manifold for a semilinear elliptic problem with a sign changing weight function. J. Differ. Equ. 193, 481-499 (2003)

[12] Drabek, P., Pohozaev, S.I.: Positive solutions for the p-Laplacian: application of the fibering method. Proc. Royal Soc. Edinburgh Sect A, 127, 721-747 (1997)

[13] Escobar, J.F.: Uniqueness theorems on conformal deformations of metrics, Sobolev inequalities, and an eigenvalue estimate. Comm. Pure Appl. Math. 43, 857$883(1990)$

[14] Hai, D.D., Schmitt, K., Shivaji, R.: Positive solutions of quasilinear boundary value problems. J. Math. Anal. Appl. 217(2), 672-686 (1998)

[15] Oruganti, S., Shi, J., Shivaji, R.: Logistic equation with the p-Laplacian and constant yeild harvesting. Abstract. Appl. Anal. 9, 723-727 (2004)

[16] Perera, K., Shivaji, R.: Positive solutions of multiparameter semipositone p-Laplacian problems. J. Math. Anal. Appl. 338(2), 1397-1400 (2008) 
[17] Tehrani, H.T.: A multiplicity result for the jumping nonlinearity problem. J. Differ. Equ. 118(1), 272-305 (2003)

[18] Tehrani, H.T.: On indefinite superlinear elliptic equations. Cal. Var. 4, 139-153 (1996)

[19] Tolksdorf, P.: Regularity for a more general class of quasilinear elliptic equations. J. Differ. Equ. 51, 126-150 (1984)

[20] Wu, T.F.: On semilinear elliptic equations involving concave-convex nonlinearities and sign-changing weight function. J. Math. Anal. Appl. 318, 253-270 (2006)

[21] Wu, T.F.: A semilinear elliptic problem involving nonlinear boundary condition and sign-changing potential. Electron. J. Differ. Equ. 131, 1-15 (2006)

[22] Wu, T.F.: Multiplicity results for a semilinear elliptic equation involving signchanging weight function. Rocky Mountain J. Math. (in press)

G. A. Afrouzi, S. H. Rasouli

Department of Mathematics, Faculty of Basic Sciences,

Mazandaran University,

Babolsar, Iran

e-mail: afrouzi@umz.ac.ir

S. H. Rasouli

e-mail: s.h.rasouli@umz.ac.ir

Received: 04 October 2008.

Accepted: 24 May 2009. 